\title{
Scalp EEG in Temporal Lobe Epilepsy Surgery
}

\author{
Mark Sadler and Richard Desbiens
}

\begin{abstract}
Electroencephalography (EEG) with standard scalp and additional noninvasive electrodes plays a major role in the selection of patients for temporal lobe epilepsy surgery. Recent studies have provided data supporting the value of interictal and postictal EEG in assessing the site of ictal onset. Scalp ictal rhythms are morphologically complex but at least one pattern (a five cycles/second rhythm maximum at the sphenoidal or anterior temporal electrode) occurs in $>50 \%$ of patients and has a high predictive value and interobserver reliability for temporal lobe originating seizures. Thorough interictal and ictal scalp EEG evaluation, in conjunction with modern neuroimaging, is sufficient for proceeding to surgery without invasive recordings in some patients. Further studies are required to define the scalp ictal characteristics of mesial vs. lateral temporal lobe epilepsy.
\end{abstract}

RÉSUMÉ: L'ÉEG de surface dans la chirurgie de l'épilepsie temporale. L'électroencéphalographie (ÉEG) enregistré au moyen d'électrodes standard de surface et d'électrodes non effractives additionnelles a un rôle majeur dans la sélection des patients pour la chirurgie de l'épilepsie temporale. Des études récentes supportent la valeur de l'ÉEG interictal et postictal dans l'évaluation du site de déclenchement de la crise. Les rythmes de l'enregistrement de surface de la crise ont une morphologie complexe, mais au moins un patron (un rythme de 5 cycles/seconde enregistré par l'électrode sphénoïdale ou temporale antérieure) qu'on retrouve chez plus de $50 \%$ des patients, a une valeur prédictive et une fiabilité inter-observateur élevées dans les crises originant dans le lobe temporal. L'évaluation minutieuse par l'ÉEG de surface interictal et ictal, conjointement avec la neuroimagerie moderne sont suffisantes pour procéder à la chirurgie sans enregistrement invasif chez certains patients. D'autres études sont nécessaires pour définir les caractéristiques de l'épilepsie temporale mésiale par rapport à l'épilepsie temporale latérale, à l'enregistrement ictal de surface.

Can. J. Neurol. Sci. 2000; 27: Suppl. 1 - S22-S28

Scalp EEG telemetry is an electrophysiologic technique that involves continuous recording of EEG and patient behavior (image and sound) over prolonged periods. In the context of epilepsy surgery, this investigation is used to assist in defining the ictal and interictal epileptogenic cortical areas by obtaining EEG signals with synchronized assessment of the patient's behavior during recorded seizures. In optimum circumstances for surgical intervention there will be a congruence of the EEG with clinical, radiological, and neuropsychological data. This review will focus on the use of scalp EEG in the evaluation of candidates for temporal lobe epilepsy surgery.

\section{SOME TECHNICAL CONSIDERATIONS}

The International Standard ten-twenty electrode system continues to be the most widely accepted method of measurement and application of EEG scalp electrodes. ${ }^{1}$ Other systems describing additional electrode positions have been proposed. ${ }^{2}$ A variety of additional "nonstandard" electrodes have been described to more completely evaluate the EEG abnormalities in temporal lobe epilepsy (TLE). These include sphenoidal (SP), nasopharyngeal (NP), "anterior temporal" (AT or sometimes referred to as T1/T2 electrodes), ${ }^{3}$ "minisphenoidals", 4 and surface electrodes applied over the mandibular notch $(\mathrm{MN})^{5}$ or zygoma. Of these, NP electrodes have not consistently demonstrated superior sensitivity for spike detection compared to the other non-standard electrodes. ${ }^{5,6-7}$ In addition, NPelectrodes are uncomfortable for the patient and are not suitable for long term recording. SP, MN, and AT electrodes will record spikes not detected by standard electrodes; in one study $^{5}$ the standard electrodes detected only $58 \%$ of all spikes. SP spikes are almost always detected by MN (or AT) electrodes and their amplitudes are only slightly less than SP spikes.,

From the Queen Elizabeth II Health Sciences Centre, Halifax, N.S. (MS) and Hôpital de l'Enfant-Jésus, Quebec City, Quebec (RD).

Reprint requests to: Mark Sadler, Room 3829, New Halifax Infirmary, Queen

Elizabeth II Health Sciences Centre, 1796 Summer St., Halifax, Nova Scotia, Canada B3H $3 \mathrm{~A} 7$ 
Kanner et $\mathrm{al}^{9}$ demonstrated superior results of sphenoidal electrodes only if they were inserted under fluoroscopic guidance. Recording with multiple nonstandard electrodes simultaneously to obtain a more comprehensive evaluation of the electrical field has been recommended. ${ }^{10}$

Few studies have evaluated the ictal (as opposed to interictal) merits of these electrodes. In an ictal study comparing sphenoidal and minisphenoidal electrodes, Wilkus ${ }^{11}$ found that these electrodes produced virtually equivalent findings. Similarly, Krauss et $\mathrm{al}^{12}$ found no substantial difference between sphenoidal and cheek electrodes for localizing ictal activity.

Relatively few studies have addressed the issue of montage design for interictal and ictal temporal lobe epilepsy abnormalities. The ability to perform off-line montage reformatting with modern digital EEG technology diminishes the importance of initial montage selection. Ives et al $^{13}$ studied ictal EEGs in patients with sphenoidal electrodes by using a coronalsphenoidal montage compared to an anterior-posterior temporal montage. The montage containing the sphenoidal electrodes led to an earlier detection (usually by $>5$ seconds) and detected (in $19 \%$ of seizures) ictal changes not apparent with the anteriorposterior montage.

\section{INTERICTALABNORMALITIES}

\section{Epileptiform abnormalities}

The classic interictal EEG abnormality of partial complex seizures of temporal lobe origin is a spike (or sharp wave) that has an electronegative peak over the anterior temporal region (electrodes F7/F8) when standard EEG electrode positions are used. ${ }^{14}$ One study of a relatively "pure culture" of medial temporal lobe epilepsy demonstrated the anterior temporal location of the interictal discharges in $94 \%$ of 64 patients. ${ }^{15}$ Computer field mapping techniques show that the peak field of anterior temporal discharges is anterior and inferior to the standard 10-20 electrode positions and accounts for the sensitivity of the additional electrodes described above. ${ }^{16}$

The precise anatomic generators of discharges detected by scalp and sphenoidal electrodes are unclear. In a study using simultaneous surface and intracranial recording, electronegative sphenoidal spikes were recorded when electropositive hippocampal spikes were obtained; negative hippocampal spikes were spatially restricted and not detected at the sphenoidal position. ${ }^{17}$ Although most patients with sphenoidal maximum spikes had hippocampal originating seizures, this interictal pattern was also noted in two of three patients with temporal neocortical foci and three of six patients without localized seizure onset, including one patient with an orbital frontal focus. ${ }^{17}$ Thus, while sphenoidal maximum spikes are sensitive to mesial TLE, they are not specific.

Studies, using dipole modeling techniques and magnetoencephalography in TLE patients, have suggested two sources of surface recorded interictal spikes: (a) a vertical dipole consisting of electronegativity at the sphenoidal electrode with widespread (volume conducted) electropositivity at the vertex, and (b) a lateral temporal neocortical source that is not generated by volume conduction but rather represents synaptic propagation from mesial to lateral structures (or vice versa). ${ }^{18-20}$

However, other investigators have questioned the validity of the source model techniques and do not believe that these methods can reliably distinguish volume conduction from neurophysiologic propagation. ${ }^{21}$

Spikes associated with TLE are not necessarily confined to one temporal lobe. At least $25-33 \%$ of patients with this form of epilepsy will demonstrate independent bitemporal discharges. ${ }^{14,22}$ The substantial recording time of patients on telemetry yields more sampling, thus it would not be surprising if publications from centres using telemetry data reported a higher percentage of bilateral abnormalities. A surgical series found $42 \%$ of patients to have bilateral independent discharges. ${ }^{15}$ The presence of independent bitemporal spikes does not exclude the possibility of successful epilepsy surgery. So et $\mathrm{al}^{23}$ described their results from a series of 48 patients with independent bitemporal spikes, temporal lobe surgery, and a minimum of two year postoperative follow-up. Among these patients, 14 were seizure-free, three had less than three seizures/year, 22 had $>50 \%$ improvement in seizure frequency, and seven were not improved. Chung et $\mathrm{al}^{24}$ correlated the degree of scalp lateralization of interictal spikes with the results of temporal lobectomy; $92 \%$ of patients with $>90 \%$ of spikes confined to one side had a good surgical outcome whereas only $50 \%$ of patients with $<90 \%$ lateralization had a good outcome. ${ }^{24}$ Holmes et $\mathrm{al}^{25}$ demonstrated that patients with independent bitemporal interictal spikes (all of whom also had intracranial monitoring) could have successful surgery but the factors that were predictive of a seizure-free outcome were the occurrence of at least two of the following: MRI scan abnormality concordant with the side of surgery, $100 \%$ of intracranially recorded seizures from one side, and a history of febrile convulsions.

Sleep increases the spike frequency in $\mathrm{TLE}^{26}$ and spike foci unapparent during wakefulness may be found. ${ }^{27}$ It has been suggested that spike discharges recorded during REM sleep and wakefulness are more predictive of the site of seizure origin than spikes recorded during non-REM sleep. ${ }^{27}$

The relationship of the location and frequency of interictal spikes to the site of ictal onset has been contentious. A recent review $^{22}$ noted that "it seems intuitively obvious that patients with a unifocal or very strongly predominant interictal temporal spike or sharp wave have a strong likelihood of ictal onset from the same temporal region. Practical experience and accumulated evidence supports this presumption." However, these authors cite studies that suggest "a predominant lateralized surface interictal finding will incorrectly predict the temporal lobe of predominant ictal origin in $20-40 \%$ of cases and ... the presence of a unifocal surface temporal interictal epileptiform abnormality does not exclude the possibility of bilateral independent ictal onsets as documented by subsequent intracranial study". ${ }^{22}$

Conversely, several studies in this decade have demonstrated a strong correlation between the side of ictal onset and the side with a preponderance of interictal spikes. ${ }^{24,28-30}$ Blume et $\mathrm{al}^{28}$ found that 52 out of 56 patients (93\%) with seizures arising from one temporal lobe had interictal spikes exclusively or predominantly ipsilateral to seizure origin. In a companion study involving a different group of patients, 99/104 patients (95\%) with temporal spikes on four or more awake recordings had most or all seizures onset ipsilateral to most spikes, including 79/80 patients $(99 \%)$ of those with a side vs. side spike ratio $>3 .{ }^{28}$ Holmes et $\mathrm{al}^{30}$ reviewed their experience of 98 patients with

Suppl. 1 - S23 
complex partial seizures of whom 59 had spikes predominantly (defined as $>75 \%$ of all spikes) localized to a single unilateral region. Among these 59 patients, 48 had ictal EEGs that were interpretable and localized to one region; 39 of the $48(81 \%)$ patients had all seizures arise from the expected area. In the 48 patients were 23 who had spikes exclusively from one area and the seizures arose from the expected area in 22 of the 23 cases $(95 \%)$. Therefore, recent publications support the value of the site of maximum interictal spike frequency for predicting the site of ictal onset.

Patients are occasionally encountered who, in addition to their focal EEG abnormalities, display bisynchronous spike waves. $^{31-32}$ The mechanism of production of these diffuse waves is unclear but their presence should not deter the search for a surgically resectable focus. ${ }^{32}$

\section{Non-epileptiform abnormalities}

In general, focal slow frequency wave forms (theta and delta) are "nonspecific" findings and are seen in a variety of processes, including epilepsy. Reiher et $\mathrm{a}^{33}$ suggested that temporal intermittent rhythmic delta activity (TIRDA) is a highly specific and accurate interictal indicator of temporal lobe seizures. This particular EEG pattern has recently been validated in a surgical series. ${ }^{34}$

Focal polymorphic delta in patients with established epilepsy should not be ignored when assessing the side of ictal onset. Gambardella et $\mathrm{al}^{35}$ found trains of polymorphic delta in $>90 \%$ of 56 patients with MRI demonstrated hippocampal atrophy and TLE. The delta lateralized to the side of atrophy with accuracy equal to spikes and was never discordant from spikes. The authors concluded that intermittent focal delta is a reliable indicator of the side of the epileptogenic focus and reflects the epileptogenic process rather than the structural pathology. ${ }^{35}$ In Blume's ${ }^{28}$ study of interictal indices, 46 out of 56 patients $(82 \%)$ with seizures exclusively from one temporal lobe had unilateral (or predominantly unilateral) delta that was ipsilateral to the side of ictal onset and never falsely lateralized the ictal onset. The predominant side of seizure origin correlated with the lateralized delta in $90-100 \%$ of patients in the companion study of 156 TLE patients (with various side-to-side ratios of delta). ${ }^{28}$

\section{ICTAL EEG}

\section{Ictal and postictal scalp rhythms}

Many epilepsy surgical centres have published their preoperative protocols and the majority indicate a reliance on ictal EEG as an important component of the surgical decision making process. ${ }^{36}$ However, the sensitivity, specificity, and interobserver reliability of scalp ictal EEG for localization of the epileptogenic zone has been disputed. Recognition of the limitations of scalp ictal recordings led to the development of invasive (intracranial) recording techniques in the 1960s and 1970s and much of what is now known of the electrophysiology of temporal lobe epilepsy was obtained from these techniques. ${ }^{37}$ The combination of this knowledge with remarkable progress in neuroimaging has led to a substantial decrease in the need for invasive EEG. ${ }^{37}$

Klass ${ }^{14}$ credits Gastaut for the observation that "the attacks of psychomotor epilepsy are almost equally complex from an electroencephalographic and clinical point of view, and they cannot be reduced to a single mode of expression." The multiple
EEG expressions of temporal lobe originating seizures are likely a consequence of several factors, some of which may be interdependent. These factors could include the precise focus of ictal onset (e.g. mesial vs. lateral temporal lobe), the underlying neuropathology (e.g. mesial temporal seizures from hippocampal sclerosis vs. a neoplasm in the same anatomic region), and variable routes of propagation.

Examples of the complexities of propagation were illustrated by Lieb et $\mathrm{al}^{38}$ who studied propagation patterns of mesial temporal lobe seizures with ictal depth recordings in 24 patients. These authors, "despite attempts to be parsimonious," required nine different seizure patterns to encompass their findings. They suggested factors that may alter propagation (and therefore the EEG expression of seizures) include the state of the patient (wake vs. sleep), antiepileptic drugs, and whether the patient had unifocal vs. multifocal epilepsy.

The initial EEG change of a scalp-recorded partial seizure may be a diffuse attenuation of background activity, but this occurs in only $11-25 \%$ of seizures ${ }^{14,39}$ and has localizing or lateralizing features in a minority of cases. $^{22}$ In a small percentage of patients a seizure may appear to start, stop, and begin again. This "start-stop-start" phenomenon was originally described in subdurally recorded seizures ${ }^{40}$ but has been observed in $13 \%$ of patients with scalp ictal EEG. ${ }^{41}$ The initial start typically has a more restricted field than the second start. If the initial start is overlooked, the restart may be misinterpreted as the actual seizure onset and be considered nonlocalizing while the initial start is typically very focal and localizing. ${ }^{41}$

After this initial change, a variety of EEG waveforms are seen. In a study of the EEG morphology of partial seizures Blume et $\mathrm{al}^{39}$ found the initial features (other than attenuation) to be sinusoidal waves $(47 \%)$, repetitive epileptiform potentials $(39 \%)$, or both $(15 \%)$. As the seizures evolved, there was a change in frequency of these phenomena which either increased, decreased, or went in either direction in different EEG channels; only later in the seizures was there a decrease in frequency. Note is made that not all patients in this study had temporal lobe originating seizures.

A lateralized ictal change, variably described as "rhythmic 5 to $10 \mathrm{~Hz}$ sharp activity", ${ }^{14}$ a "5 cycles/second (or faster) rhythm maximum at a sphenoidal or temporal electrode position", 42 or "rhythmic theta-alpha" 43 occurs within $30-40$ seconds of seizure onset in $52-80 \%$ of patients. ${ }^{15,42-44}$ This pattern has high specificity for TLE (see further discussion in the section of this paper on "Accuracy of scalp ictal recordings").

Postictal changes are important for seizure lateralization. Kaibara and Blume, ${ }^{45}$ in a study of scalp recorded seizures (of which $42 / 51$ began in the temporal lobe), found postictal changes in $69 \%$. These changes included regional delta, regional attenuation, and activation of spikes. Postictal change, whatever its nature, appeared principally or exclusively ipsilateral to the side of seizure onset in all cases. In the Williamson et $\mathrm{al}^{15}$ study of patients rendered seizure-free after a temporal lobectomy, $67 \%$ of patients had lateralized postictal slowing that was concordant to the side of seizure origin in $100 \%$ of instances. Similarly, Walczak et $\mathrm{al}^{43}$ found that when postictal changes were present and could be lateralized (43-66\% of patients) these findings were concordant with the side of seizure onset in 96$100 \%$ of patients at least two years seizure-free after surgery. 


\section{Scalp-intracranial ictal relationships}

It has been known for many years that scalp EEG incompletely records many aspects of intracranial activity. ${ }^{46}$ Lieb et al, ${ }^{47}$ using a combination of surface and depth electrodes in patients with temporal lobe epilepsy, demonstrated that a large proportion of seizures initially seen with depth recordings produce no visible changes in surface EEG recordings or produce bilateral abnormalities in the surface EEG which are not indicative of the side from which depth recorded seizure activity was initiated. Only $19 \%$ of auras and $10 \%$ of subclinical seizures were accompanied by scalp EEG changes. A shortcoming of this study was the limited number of recording channels, such that highly detailed scalp-surface relationships could not be performed.

The most comprehensive systematic study of scalpintracranial ictal EEG findings with temporal lobe epilepsy has recently been published. ${ }^{48}$ Pacia and Ebersole reported their findings from 24 patients (with 110 recorded seizures) with TLE. This study is unique for two reasons : (a) inclusion of patients with seizures arising from both mesial and lateral temporal structures and, (b) the extensive EEG data collection: the EEG was obtained from sixty-four channels of digital EEG (allowing off-line manipulation) recorded simultaneously from scalp electrodes, mesial temporal depth probes, subdural strip electrodes, and (in two patients) subdural grids. Their findings are summarized as follows.

Patients with seizures beginning at the hippocampal contacts of the depth electrode had ictal rhythms recorded from the mesial subdural strip electrodes. Simultaneous scalp recordings showed either no change or a diffuse disruption of background; only when the seizure spread to the basal and inferolateral subdural contacts did a 6-7 cycles/second rhythm appear in the inferior and standard scalp electrodes. The authors concluded that a scalp recorded 5-9 cycles/second rhythm is highly associated with hippocampal onset seizures but is not a direct manifestation of hippocampal ictal activity. This characteristic rhythm (see discussion by Risinger et $\mathrm{al}^{27}$ and Walczak et al, ${ }^{43}$ below) appears to reflect recruitment of adjacent temporal cortex. Pacia and Ebersole $^{48}$ called this a "type 1 A seizure." This rhythm appears to be dependent not only on the spread of the seizure to temporal cortex but involves cortico-hippocampal interactions because seizures initiated in the neocortex did not demonstrate this scalp pattern until the hippocampus became involved. ${ }^{44}$

Seizures confined to the mesial-basal cortex ("type 1B") produce a different scalp rhythm that is manifest on the scalp as a 5 cycles/second rhythm maximally expressed, perhaps unexpectedly, at the vertex. This rhythm is of opposite polarity from that seen with the intracranial basal electrodes and "although seemingly unlateralized, is in fact quite localizing because of the particular cortical orientation required for its generation". ${ }^{48}$

"Type 2" seizures were highly associated with temporal neocortical onsets. The intracranial correlate of this seizure type was a fast frequency (in the beta frequency range or higher) and was either focal or regional in onset; such high frequency changes were never seen with scalp recordings in this study. The corresponding scalp recordings consisted of a high voltage, irregular, delta frequency pattern. Interestingly, three patients demonstrated this "type 2" pattern but had mesial temporal onset of seizures; two of these three patients had "atypical" spread of their seizures because propagation to the opposite temporal lobe occurred very rapidly ( $<5$ seconds). The third patient also had a seizure onset different than the classic depth recorded seizures of hippocampal onset in that a diffuse seizure onset affecting all the depth contacts simultaneously was recorded.

"Type 3" seizures had scalp EEG characterized by changes but not typical rhythmic patterns or other features characteristic of an ictus; such patients had seizures onsetting in either the hippocampus or the neocortex. It was thought that the reason for failing to record ictal rhythms at the scalp in these patients was that the seizures were confined to the hippocampus or there was insufficient synchrony of cortical areas.

\section{Accuracy of scalp ictal recordings}

A crucial question in the surgical management of temporal lobe epilepsy is whether scalp recorded seizures can accurately lateralize (i.e. identify the correct hemisphere) and localize (i.e. identify the correct lobe) of ictal onset. Potential difficulties with scalp recordings include an inability to interpret the EEG because of muscle and movement artifacts, a paucity of unequivocal changes, false lateralization, and interobserver disagreement. ${ }^{44}$

Spencer et $\mathrm{al}^{44}$ were the first to study the interobserver reliability in localizing ictal scalp EEGs. In this study, 144 scalp ictal EEGs from 54 patients (who had both scalp and depth EEG) were interpreted by three blinded electroencephalographers (EEGers). Depth recorded seizures were considered the "gold standard" for comparison. Among the 27 patients with temporal lobe epilepsy, accuracy of scalp lateralization was $57-60 \%$ but lateralization contralateral to the depth recorded seizures occurred in $3-17 \%$ of cases. The kappa statistic (a measurement of index of agreement, corrected for chance) was calculated to measure the reliability of EEG interpretation between pairs of EEGers. Reliability for lateralization was "fair to good"; reliability for identification of lobe of seizure onset was "poor to fair." When scalp EEGs that were unlocalizable were excluded, there was "good to excellent" agreement among the EEGers for the temporal cases. The authors concluded that more formal criteria were needed before scalp ictal records could be used reliably or accurately for localization. Criticisms of this study include the lack of sphenoidal or other nonstandard electrodes and the limited number of EEG recording channels.

Risinger et $\mathrm{al}^{42}$ attempted to define more formal criteria for scalp localization of temporal lobe epilepsy. They assessed the reliability and accuracy of scalp/sphenoidal recordings for ictal localization by retrospectively analyzing 706 noninvasive ictal recordings from 110 patients who subsequently underwent invasive depth EEG. The hypothesis in this study was that an easily identified 5 cycles/second (or faster) rhythm maximum at one sphenoidal or temporal electrode position early in the course of a seizure was predictive of an ipsilateral temporal lobe onset on depth examination. Fifty-two percent of patients displayed this pattern and of these, $82 \%$ had an ipsilateral temporal lobe onset determined by depth recordings. In those patients who demonstrated exclusively "focal" scalp recordings, the predictive value of this pattern increased to $94 \%$ but fell to $67 \%$ in patients with a mixture of focal and nonfocal recordings. Among three unblinded EEGers the interrater reliability for right focal vs. left focal vs. nonfocal was "excellent" with a kappa statistic of 
$>0.83$. However, in $10 / 57(18 \%)$ of all cases the presence of the 5 cycles/second rhythm was misleading. The errors were of two types: most commonly (eight cases), the rhythm predicted an onset in a temporal lobe that depth recordings subsequently failed to demonstrate an onset in either temporal lobe (i.e. an extratemporal originating seizure). The second type of error was false lateralization (two cases). The authors concluded that the 5 cycles/second rhythm was misleading in only a minority of cases but cannot be used in isolation for definite ictal localization.

More recently Walczak et $\mathrm{al}^{43}$ published a similar study but used outcome from epilepsy surgery as the "gold standard" of the accuracy of scalp ictal EEG in identifying the epileptogenic zone. Three blinded EEGers retrospectively assessed 137 ictal EEGs from 35 patients with temporal resections and five patients with extratemporal resections, all of whom were at least two years seizure-free. Three EEG features were analyzed: (a) activity at seizure onset, (b) rhythmic waves in the alpha or theta frequency range lasting at least 10 seconds and occurring within 40 seconds of seizure onset (similar to the rhythm described by Risinger et al, ${ }^{42}$ and (c) postictal findings. Overall, the EEGers predicted the correct side of seizure onset in 76-83\% of the 119 seizures in the temporal cases (kappa statistic of $0.59-0.69$, indicating a clinical significance of "fair to good"). When the analysis was restricted to only seizures that had lateralizing features, the accuracy of lateralization was 93-98\% (kappa of 0.85-0.96). When individual EEG features were analyzed, the alpha-theta frequency pattern and postictal slowing were more accurate than the activity at seizure onset. Specifically, the rhythmic alpha-theta pattern (found in $64-78 \%$ of all temporal seizures) was $64-76 \%$ accurate for all temporal lobe seizures and when lateralization was possible it was $97-98 \%$ accurate. Post ictal findings were only $43-64 \%$ accurate for all temporal lobe seizures but when lateralization was possible (49-63\% of seizures) it was $96-100 \%$ accurate. The errors of lateralization were analyzed as follows: the three EEGers made a total of ten lateralization errors among seven seizures in four patients; 8 out of 10 errors occurred in seizures in which only one of the three EEG features studied was lateralizable; no lateralization errors occurred in the 76 seizures that contained all three EEG features that could be lateralized.

The few cases of false lateralization reported in the literature in detail ${ }^{49-50}$ have emphasized the role of gross focal lesions in altering EEG ictal patterns to create the impression of a seizure onset contralateral to the true side.

Sperling et $\mathrm{al}^{51}$ published, in 1992, a noninvasive protocol for anterior temporal lobectomy that incorporates clinical ictal features (recorded with videotelemetry), interictal and ictal scalp EEG (with sphenoidal electrodes), MRI, neuropsychology (including bilateral intracarotid amobarbital testing), and the thiopental activation test (a positive test was a failure of EEG beta rhythms to develop unilaterally when the patient received intravenous thiopental). Patients subjected to this protocol included those with medically resistant epilepsy, ictal clinical features typical of temporal lobe originating seizures, and interictal EEG spikes from one or both temporal lobes. Exclusion criterion were patients suspected of extratemporal epilepsy based on clinical features, extratemporal interictal spikes, or an imaging study demonstrating an extratemporal lesion. An important aspect to appreciate in this protocol was that a "positive" MRI refers only to a cyst or tumor; hippocampal or temporal lobe atrophy was not used as a protocol criterion. Patients meeting the inclusion criteria were initially categorized by the MRI scan. A temporal lobectomy was offered if the MRI disclosed a temporal lesion, the interictal or ictal EEG was congruent, and if the intracarotid amobarbital demonstrated preservation of memory in the contralateral hemisphere. If the MRI was negative, a different set of criteria were used. "Primary test criteria" included state independent (awake and sleep) spikes maximal at the sphenoidal electrode and $>90 \%$ unilateral preponderance or initial seizure EEG rhythms maximal at a sphenoidal electrode within 30 seconds of seizure onset. "Secondary test criteria" were state dependent (sleep only) spikes maximum at the sphenoidal electrode with $>90 \%$ unilateral preponderance, interictal focal EEG slowing at the sphenoidal electrode $>50 \%$ of the recording time, localized beta defect with thiopental, or unilateral memory impairment with the intracarotid amobarbital test. A temporal lobectomy was offered if either two primary criteria and one secondary criterion or one primary and three secondary criteria were met. Intracranial monitoring was done if there were contradictory findings, an insufficient number of positive tests, or if interictal spikes were maximum at T3/4 compared to SP1/2 electrodes. Among 103 patients evaluated for temporal lobe surgery between 1986-1989, 51 met the noninvasive protocol criteria and had surgery. Of these operated patients (follow-up of at least 1.75 years), $80 \%$ were seizure-free, $10 \%$ had less than 3 seizures/year or have strictly nocturnal seizures, and $10 \%$ had a $>80 \%$ seizure reduction. The authors concluded that this was a safe and effective protocol for evaluating patients with noninvasive EEG. In addition, upon reviewing the contribution of the various tests, Sperling et $\mathrm{al}^{51}$ found that the thiopental activation procedure failed to provide unique information and could be omitted.

We are not aware of any other studies that have studied a noninvasive protocol in this fashion. It is likely that most epilepsy surgery centres in the 1990s give considerable weight to the MRI findings of temporal lobe atrophy and/or the typical MRI features of mesial temporal sclerosis, neither of which was used by Sperling et al. The number of structural and functional noninvasive investigations described for presurgical evaluation in epilepsy has grown considerably. These include interictal and ictal functional MRI (fMRI), volumetric MRI, magnetic resonance spectroscopy (MRS), interictal and ictal single photon emitted computed tomography (SPECT), interictal and ictal positron emitted tomography (PET), and magnetoencephalography. There are no prospective published protocols incorporating the results of these investigations to determine which tests provide redundant, and which provide complementary, information.

\section{HOW MANY SEIZURES NEED TO BE RECORDED?}

There is no general agreement on the number of technically adequate ictal events required to determine if surgery can proceed without invasive recordings but attempts should be made to obtain at least one sample of all seizure types in the individual case. ${ }^{22}$ An exception can be made for patients with secondarily generalized seizures, providing there is good documentation that these attacks are preceded by the patient's typical partial complex

Suppl. 1 - S26 
seizures. $^{22}$ There are no prospective studies to answer the question of how many seizures should be recorded.

Sirven et al ${ }^{52}$ retrospectively studied 734 seizures from 166 patients with suspected TLE to determine the reproducibility of seizures with scalp EEG. Not surprisingly, patients with unilateral interictal spikes were much more likely to have all seizures "concordant" (defined as all seizures localized to one temporal lobe or lateralized to one hemisphere) than patients with bilateral interictal spikes. Among the group of patients with "conflicting" seizures (defined as some seizures localized to the right temporal lobe or hemisphere and some localized/lateralized to the left), all patients demonstrated the conflicting seizures by the fourth recorded seizure. These results were similar to those of Blum ${ }^{53}$ who determined, using a statistical modeling technique, that five recorded seizures were required to have a $95 \%$ chance of avoiding a conflicting seizure and only four seizures were needed if all interictal spikes were unilateral.

Haut et $\mathrm{al}^{54}$ suggested, from a study of telemetry patients with independent bilateral seizure onsets, that seizures occurring within eight hours are likely to come from the same side. Therefore, clustered seizures should not be given the same weight as seizures widely separated in time. In this study, 3 out of 14 patients required more than five recorded seizures before independent bilateral onsets could be documented.

\section{SUMMARY AND CONCLUSIONS}

Scalp derived EEG has a major role in selecting patients for temporal lobe epilepsy surgery and in determining the necessity for invasive electrode studies. In conjunction with all other available noninvasive data, careful evaluation of interictal EEG features, ictal characteristics, and postictal changes allow the selection of many patients for surgery without resorting to invasive electrodes.

In practice, using the "basic" investigations available in epilepsy surgery programs, surgery can be offered to the "ideal" patient demonstrating the following congruence of data:

- Clinical features of the seizures compatible with seizures of temporal lobe origin. There should not be features suggesting an extratemporal onset (e.g. visual or hemiclonic phenomena early in the ictus);

- Interictal spikes with a field distribution maximum at anterior temporal electrodes (F7/8, mandibular notch, "minisphenoidal," or sphenoidal) or anterior-midtemporal electrodes; no or only rare posterior temporal or extratemporal spikes;

- Interictal spike frequency predominantly unilateral (at least $75 \%$ of all spikes);

- Unilateral temporal focal slowing of EEG background rhythms;

- Scalp ictal recordings with ictal rhythms noted at the anterior temporal electrodes early (within 30 seconds of seizure onset) and ipsilateral lateralized postictal temporal polymorphic delta rhythms. We typically do not require more than three to five recorded seizures if other data (clinical, interictal EEG, MRI, neuropsychology) are congruent to one temporal lobe;

- MRI demonstrating a structural lesion (including mesial temporal sclerosis) ipsilateral to EEG abnormalities;

- Neuropsychology (including intracarotid amytal) suggesting deficits in the temporal lobe under consideration of resection and sufficient memory function in the contralateral temporal lobe.
There are no absolute guidelines for patients who deviate from the "ideal" situation described above. Some examples are included to describe how we approach selected situations. In general, we record more seizures (e.g. 10-15) from patients who demonstrate independent bitemporal spikes with less than 75$90 \%$ laterality; we will proceed to temporal lobectomy if the ictal rhythms are clearly unilateral and congruent with an MRI abnormality. We would consider a temporal lobectomy, without invasive recordings, if $>75 \%$ of independent bitemporal seizures originate from a side that is congruent with an ipsilateral MRI lesion. In these situations, the neuropsychology data may "tip the balance" in favour of proceeding to surgery without invasive EEG or suggest the requirement for more intensive electrophysiology.

Conversely, we would suggest invasive recordings for patients with partial complex seizures (and clinical features of temporal lobe epilepsy) who demonstrate:

- Independent bitemporal spikes and seizures with no apparent MRI lesion or bilateral temporal MRI structural abnormalities;

- Interictal EEG data and seizure onsets apparently contralateral to a structural MRI lesion.

TLE can be considered on a spectrum from patients with "strictly unilateral" TLE (who have the potential of surgical cure of their disease) to patients with "approximately equal" TLE (who are very unlikely to be cured of their epilepsy with surgery). In between these extremes are patients who can obtain varying degrees of seizure control with resective surgery. Scalp EEG, in conjunction with other modalities, can be used to select surgical candidates and define those patients for whom invasive recordings are mandatory.

\section{REFERENCES}

1. Jasper H. The ten-twenty electrode system of the International Federation. Electroencephalogr Clin Neurophysiol 1958;10:371-375.

2. Nuwer MR. Recording electrode site nomenclature. J Clin Neurophysiol 1987;4:121-133.

3. Silverman D. The anterior temporal electrode and the ten-twenty system. Electroencephalogr Clin Neurophysiol 1960;12:735-737.

4. Laxer KD. Minisphenoidal electrodes in the investigation of seizures. Electroencephalogr Clin Neurophysiol 1984;58:127-129.

5. Sadler RM, Goodwin J. Multiple electrodes for detecting spikes in partial complex seizures. Can J Neurol Sci 1989;16:326-329.

6. Sperling MR, Engel J. EEG from the temporal lobes: a comparison of ear, anterior temporal, and nasopharyngeal electrodes. Ann Neurol 1985;17:510-513.

7. Sperling MR, Mendius JR, Engel J. Mesial temporal spikes: a simultaneous comparison of sphenoidal, nasopharyngeal, and ear electrodes. Epilepsia; 1986:27:81-86.

8. Binnie CD, Marston D, Polkey CE, et al. Distribution of temporal spikes in relation to the sphenoidal electrode. Electroencephalogr Clin Neurophysiol 1989;73:403-409.

9. Kanner AM, Ramirez L, Jones JC.The utility of placing sphenoidal electrodes under the foramen ovale with fluoroscopic guidance. $\mathrm{J}$ Clin Neurophysiol 1995;12: 72-81.

10. Morris HH, Luders H, Lesser RP, et al. The value of closely spaced scalp electrodes in the localization of epileptiform foci: a study of 26 patients with complex partial seizures. Electroencephalogr Clin Neurophysiol 1988;70:105-109.

11. Wilkus RJ, Thompson PM, Vossler DG. Quantitative comparison of ictal EEG from sphenoidal, minisphenoidal, anterior temporal, and midtemporal electrodes. Epilepsia 1991;32(suppl3):100-101.

12. Krauss GL, Lesser RP, Fisher RF, et al. Anterior "cheek" electrodes are comparable to sphenoidal electrodes for the identification of 
ictal activity. Electroencephalogr Clin Neurophysiol 1992;83:333-338.

13. Ives JR, Drislane FW, Schacter SC, et al. Comparison of coronal sphenoidal versus anteroposterior temporal montage in the EEG recording of temporal lobe seizures. Electroencephalogr Clin Neurophysiol 1996; 98:417-421.

14. Klass D. Electroencephalographic manifestations of complex partial seizures. In: Penry JK and Daly DD (Eds), Advances in Neurology. Vol. 11. Complex Partial Seizures and Their Treatment. New York: Raven Press. 1975:113-140.

15. Williamson PD, French JA, Thadani VM, et al. Characteristics of medial temporal lobe epilepsy: II. Interictal and ictal scalp electroencephalography, neuropsychological testing, neuroimaging, surgical results, and pathology. Ann Neurol 1993;34: 781-787.

16. Sadler RM, Lemieux JF, Blume WT. Potential fields of anterior temporal spikes. Electroencephalogr Clin Neurophysiol 1984;58:P47-P48 (abstract).

17. Marks DA, Kratz A, Booke J, et al. Comparison and correlation of surface and sphenoidal electrodes with simultaneous intracranial recording: an interictal study. Electroencephalogr Clin Neurophysiol 1992;82:23-29.

18. Baumgartner C, Lindinger G, Ebner A, et al. Propagation of interictal epileptic activity in temporal lobe epilepsy. Neurology 1995;45:118-122.

19. Sutherling WW and Barth DS. Neocortical propagation in temporal lobe spike foci on magnetoencephalography and electroencephalography. Ann Neurol 1989;25:373-381.

20. Ebersole JS. EEG dipole modeling in complex partial epilepsy. Brain Topogr 1993;5:419-423.

21. Alarcon G, Guy CN, Binnie CD, et al. Intracerebral propagation of interictal activity in partial epilepsy: implications for source localisation. J Neurol Neurosurg Psychiatry 1994;57:435-449.

22. Quesney LF, Risinger MW, Shewmon DA. Extracranial EEG evaluation. In: J. Engel, Jr. (ed), Surgical Treatment of the Epilepsies, second edition. New York: Raven Press. 1993: 173-195.

23. So N, Olivier A, Andermann F, Gloor P, Quesney LF. Results of surgical treatment in patients with bitemporal abnormalities. Ann Neurol 1989;25:432-439.

24. Chung MY, Walczak TS, Lewis DV, Dawson DV, Radtke R. Temporal lobectomy and independent bitemporal interictal activity: what degree of lateralization is sufficient? Epilepsia 1991;32:195-201.

25. Holmes MD, Dodrill CB, Ojemann GA, Wilensky AJ, Ojemann LM. Outcome following surgery in patients with bitemporal interictal epileptiform patterns. Neurology 1997;48:1037-1040.

26. Malow BA, Kushwaha R, Lin X, et al. Relationship of interictal epileptiform discharges to sleep depth in partial epilepsy. Electroencephalogr Clin Neurophysiol 1997;102:20-26.

27. Sammaritano M, Gigli GL, Gotman J. Interictal spiking during wakefulness and sleep and the localization of foci in temporal lobe epilepsy. Neurology 1991;41:290-297.

28. Blume WT, Borghesi JL, Lemieux JF. Interictal indices of temporal lobe origin. Ann Neurol 1993;34:703-709.

29. Kanner A, Morris H, Luders H, et al. Usefulness of unilateral interictal sharp waves of temporal lobe origin in prolonged videoEEG monitoring studies. Epilepsia 1993;34:884-889.

30. Holmes MD, Dodrill CB, Wilensky AJ, et al. Unilateral focal preponderence of interictal epileptiform discharges as a predictor of seizure origin. Arch Neurol 1996;53:228-232.

31. Gabor AJ and Ajmone-Marsan C. Co-existence of focal and bilateral diffuse paroxysmal discharges in epileptics: a clinical electrographic study. Epilepsia 1969;10:453-472.

32. Sadler RM and Blume WT. Significance of bisynchronous spike-waves in patients with temporal lobe spikes. Epilepsia 1989;30: 143-146.
33. Reiher J, Beaudry M, Leduc CP. Temporal intermittent rhythmic delta activity (TIRDA) in the diagnosis of complex partial epilepsy: sensitivity, specificity and predictive value. Can J Neurol Sci 1989;16:398-401.

34. Geyer JD, Bilir E, Faught RE, Kuzniecky R, Gilliam F. Significance of interictal temporal lobe delta activity for localization of the primary epileptogenic region. Neurology 1999;52:202-205.

35. Gambardella A, Gotman J, Cendes F, Andermann F. Focal intermittent delta activity in patients with mesiotemporal atrophy: a reliable marker of the epileptogenic focus. Epilepsia 1995;36:122-129.

36. Engel J. Update on surgical treatment of the epilepsies. Neurology 1993;43:1612-1617.

37. King D, Spencer S. Invasive electroencephalography in mesial temporal lobe epilepsy. J Clin Neurophysiol 1995;12:32-45.

38. Lieb J, Dasheiff R, Engel J. Role of the frontal lobes in the propagation of mesial temporal lobe seizures. Epilepsia 1991;32:822-837.

39. Blume WT, Young GB, Lemieux JF. EEG morphology of partial epileptic seizures. Electroencephalogr Clin Neurophysiol 1984;57:295-302.

40. Blume WT, Kaibara M. The start-stop-start phenomenon of subdurally recorded seizures. Electroencephalogr Clin Neurophysiol 1993;86:94-99.

41. Atalla N, Abou-Khalil B, Fakhoury T. The start-stop-start phenomenon in scalp-sphenoidal recordings. Electroencephalogr Clin Neurophysiol 1996;98:9-13.

42. Risinger M, Engel J, Van Ness P, et al. Ictal localization of temporal lobe seizures with scalp/sphenoidal recordings. Neurology 1989;39:1288-1293.

43. Walczak T, Radtke R, Lewis D. Accuracy and interobserver reliability of scalp ictal EEG. Neurology 1992;42:2279-2285.

44. Spencer $S$, Williamson P, Bridgers S, et al. Reliability and accuracy of localization by scalp ictal EEG. Neurology 1985;35:15671575.

45. Kaibara M, Blume WT. The postictal electroencephalogram. Electroencephalogr Clin Neurophysiol 1988;70:99-104.

46. Cooper R, Winter AL, Crow HJ, et al. Comparison of subcortical, cortical, and scalp activity using chronic indwelling electrodes in man. Electroencephalogr Clin Neurophysiol 1965;18:217-228.

47. Lieb JP, Walsh GO, Babb TL. et al. A comparison of EEG seizure patterns recorded with surface and depth electrodes in patients with temporal lobe epilepsy. Epilepsia 1976;17:137-160.

48. Pacia SV, Ebersole JS. Intracranial EEG substrates of scalp ictal patterns from temporal lobe foci. Epilepsia 1997;38:642-654.

49. Sammaritano M, de Lotbiniere A, Andermann F, et al. False lateralization by surface EEG of seizure onset in patients with temporal lobe epilepsy and gross focal cerebral lesions. Ann Neurol 1987;21:361-369.

50. Engel J Jr, Crandall PH, Brown WJ. Consistent false lateralization of seizure onset with sphenoidal and scalp telemetred ictal EEG recordings in two patients with partial complex epilepsy. Electroencephalogr Clin Neurophysiol 1980;50:160P(abstract).

51. Sperling MR, O'Connor MJ, Saykin AJ, et al. A noninvasive protocol for anterior temporal lobectomy. Neurology 1992;42:416-422.

52. Sirven JI, Liporace JD, French JA, et al. Seizures in temporal lobe epilepsy. I. Reliability of scalp sphenoidal ictal recording. Neurology 1997;48:1041-1046.

53. Blum D. Prevalence of bilateral partial seizure foci and implications for electroencephalographic telemetry monitoring and epilepsy surgery. Electroencephalogr Clin Neurophysiol 1994;91:329-336.

54. Haut SR, Legatt AD, O'Dell C, et al. Seizure lateralization during EEG monitoring in patients with bilateral foci: the cluster effect. Epilepsia 1997;38:937-940. 